\title{
Impaired Psychomoter Performance with Topical Ocular and Nasal Ketotifen
}

\author{
Yoko ISHII ${ }^{* 1, * 2}$ Koichi NAKAMURA*1 \\ Naoto UEMURA*1 Kimiko TSUTSUMI*1 \\ Tsutomu KOTEGAWA*1 Shigeyuki NAKANO*1 \\ and Kazuo NAKATSUKA*2
}

(Received on July 7, 1999)

*1 Department of Clinical Pharmacology and Therapeutics, Oita Medical University

Hasama-machi, Oita 879-5593, Japan

*2 Department of Ophthalmology, Oita Medical University

\begin{abstract}
Background : Ketotifen, an anti-allergic drug with a histamine $\mathrm{H}_{1}$-receptor antagonistic component, has demonstrated sedative effects upon oral administration. The objective of our study was to investigate whether the topical use of ketotifen via the ocular and/or nasal route produced any sedative effects.

Methods : Twelve healthy young male volunteers received each of the following treatments on four different occasions in a randomized, double-blind, Latin-square, placebo-controlled design: (A) ketotifen eye drops $(1 \mathrm{mg})+$ ketotifen nasal drops (1 $\mathrm{mg}$ ) ; (B) ketotifen eye drops + placebo nasal drops ; (C) placebo eye drops + ketotifen nasal drops ; and (D) placebo eye drops + placebo nasal drops. Before and 1 and 2 hours after each treatment the random number addition test (RNAT), digit symbol substitution test (DSST), critical fusion frequency threshold (CFFT) and the visual analog scale (VAS) for subjective feeling of sedation were measured.

Results : Neither RNAT nor DSST showed significant changes after ocular and/or nasal administration of ketotifen. The co-administration of ketotifen via the ocular and nasal routes significantly reduced CFFT $(\mathrm{p}<0.05)$. VAS showed no significant changes in the subjective feeling of sedation.

Conclusion: Topical combined use of ketotifen via the ocular and nasal routes led to an impaired response on CFFT.
\end{abstract}

Key words : ketotifen, eye drops, nasal drops, impaired performance, critical fusion frequency

\section{Introduction}

Ketotifen is a potent anti-allergic drug with a histamine $\mathrm{H}_{1}$-receptor antagonistic component,

*1 大分医科大学臨床薬理学

T 879-5593 大分県大分郡㣣間町医大ヶ丘 1-1

*2 大分医科大学眼科学 which has shown sedative effects after oral administration ${ }^{1)}$. Ophthalmic and nasal solutions of ketotifen are often prescribed concurrently to treat patients with allergic conjunctivitis and rhinitis in Japan. Some outpatients have complained of sleepiness after the topical administration of ketotifen, and were conse- 
quently switched to other anti-allergic eye drops. The systemic effects of topical ketotifen in low doses have not been evaluated. We investigated whether the combined topical use of ketotifen by two different routes, ocular and/or nasal, produces any effects on the central nervous system in healthy young men using psychomotor performance tests.

\section{Methods}

\section{Subjects}

Twelve healthy male Japanese volunteers aged $21 \sim 25$ years were selected for the study according their medical history, smoking habits, results of physical and ocular examinations, routine laboratory tests and electrocardiogram. They were required to drink no alcohol on the night before each treatment and during the study session. Coffee or other beverages containing caffeine and nicotine were prohibited on study days. Medications including antihistamines, anti-allergics or neuroleptics that were likely to impair psychomotor performance were not allowed during the 7-day period prior to the study.

\section{Study Design}

The study was approved by the Institutional Review Board of Oita Medical University and all subjects gave written informed consent for participation in the study. This study was conducted using a randomized, double-blind, Latinsquare, placebo-controlled design. Each subject received each of the following treatments on four different occasions: (A) ketotifen eye drops + ketotifen nasal drops; (B) ketotifen eye drops + placebo nasal drops; (C) placebo eye drops+ketotifen nasal drops; and (D) placebo eye drops + placebo nasal drops. Each treatment regimen was separated by at least 3 days. The following preparations and doses were used in the study: (1) ketotifen eye drops (Zaditen ${ }^{\circledR}$ Ophthalmic Solution, Santen, Osaka, Japan, $0.1 \mathrm{mg}, 0.1 \mathrm{ml}$ per eye) ; (2) placebo eye drops (Artificial Tear Mytear ${ }^{\circledR}$, Senju, Tokyo, Japan, $0.1 \mathrm{ml}$ per eye) ; and/or (3) ketotifen nasal drops (Zaditen ${ }^{\circledR}$ Nasal Solution, Santen, Osaka, Japan, $0.1 \mathrm{mg})$; (4) placebo nasal drops (Otsuka-Seishoku-chu ${ }^{\circledR}$, Otsuka, Osaka, Japan, isotonic sodium chloride solution). Each subject received both eye and nasal drops in every treatment: initially one eye drop in the lower cul-de-sac of each eye, then one spray into each nostril, and again one eye drop into each eye. The same ophthalmologist applied the eye and nasal drops. The subjects were instructed not to compress the lacrimal duct with digital pressure.

\section{Psychomotor Performance Tests}

The battery of psychomotor performance tests included 3 minutes of the random number addition test (RNAT) and 90 seconds of the digit symbol substitution test (DSST), and the critical fusion frequency threshold (CFFT). Before beginning the study, the subjects were given five training sessions until they achieved a steady level of performance on all tasks to preclude learning effects.

In RNAT, the subjects were required to add two randomly arranged numbers and to provide the answer in a designated space. In DSST, the subjects were required to insert the appropriate symbol indicated by a code in the space below each randomly arranged number. In both RNAT and DSST, the subjects were asked to complete as many additions or substitutions as possible. The total number of additions or substitutions was recorded (Table 1). In CFFT, a flickering light was presented for 2 seconds at a lower frequency than the possible fusion point and the frequency was altered stepwise accord- 
Table 1 Results of Random Number Addition Test and Digit Symbol Substitution Test

\begin{tabular}{clcccc}
\hline Tests Time & & \multicolumn{4}{c}{ Treatments } \\
\cline { 3 - 6 } & & placebo & eye drops & nasal drops & co-administration \\
\hline RNAT & & & & \\
before & answer & $252 \pm 12$ & $249 \pm 9$ & $247 \pm 12$ & $240 \pm 10$ \\
& mistake & $2 \pm 1$ & $2 \pm 1$ & $3 \pm 1$ & $2 \pm 1$ \\
\multirow{2}{*}{$1 \mathrm{hr}$} & answer & $245 \pm 9$ & $239 \pm 5$ & $238 \pm 11$ & $233 \pm 9$ \\
& mistake & $2 \pm 0$ & $2 \pm 1$ & $2 \pm 1$ & $2 \pm 1$ \\
$2 \mathrm{hr}$ & answer & $249 \pm 9$ & $237 \pm 6$ & $241 \pm 10$ & $238 \pm 9$ \\
& mistake & $1 \pm 1$ & $2 \pm 1$ & $1 \pm 1$ & $3 \pm 1$ \\
DSST & & & & & \\
before & answer & $88 \pm 3$ & $88 \pm 2$ & $87 \pm 3$ & $88 \pm 2$ \\
& mistake & $0 \pm 0$ & $1 \pm 0$ & $0 \pm 0$ & $0 \pm 0$ \\
$1 \mathrm{hr}$ & answer & $103 \pm 16$ & $85 \pm 2$ & $85 \pm 3$ & $84 \pm 2$ \\
& mistake & $0 \pm 0$ & $1 \pm 0$ & $1 \pm 0$ & $0 \pm 0$ \\
$2 \mathrm{hr}$ & answer & $105 \pm 15$ & $88 \pm 2$ & $86 \pm 2$ & $86 \pm 2$ \\
& mistake & $1 \pm 0$ & $1 \pm 0$ & $0 \pm 0$ & $0 \pm 0$ \\
\hline
\end{tabular}

Data are mean values \pm standard error of duplicate determinations in 12 subjects. RNAT $=$ random number addition test, DSST $=$ digit symbol substitution test Time $=$ time after treatments

ing to the pattern of response. The threshold was defined as the highest frequency at which $50 \%$ or more of the last 25 responses were considered to flicker. On the visual analog scale (VAS), the subjects were instructed to mark a short rating scale comprised of a series of 100 $\mathrm{mm}$ lines at the point which corresponded to their present feeling related to sedation.

The assessments and extremes of the rating scale are shown in Table 2. The study session began at 18:00 on each treatment day. Thirty minutes after the subjects ingested a light snack, each subject practiced once to become familiar with the study procedures. They were not allowed to eat or drink during the study session on treatment days. Before and 1 and 2 hours after treatment, we measured RNAT, DSST and CFFT. VAS for subjective feelings of sedation was also measured after each study procedure.

\section{Statistical Analysis}

Data are presented as mean values \pm standard error in Table 1 and 2 and as the mean with a standard error bar in Fig. 1. Differences from baseline values on 4 treatment days were analyzed using ANOVA (analysis of variance). For statistical analysis of drug effects we utilized ANOVA with repeated measurements and Fisher's protected least significant difference using the Super ANOVA computer program (Abacus Concepts, Inc., Berkeley, CA, U.S. A.). Statistical significance of the differences was determined with $\mathrm{p}<0.05$ being the minimal level of significance accepted.

\section{Results}

No subject reported any adverse effects in the study. The results of RNAT and DSST are shown in Table 1 . Neither RNAT nor DSST showed significant changes after drug administration. Only the co-administration of the 


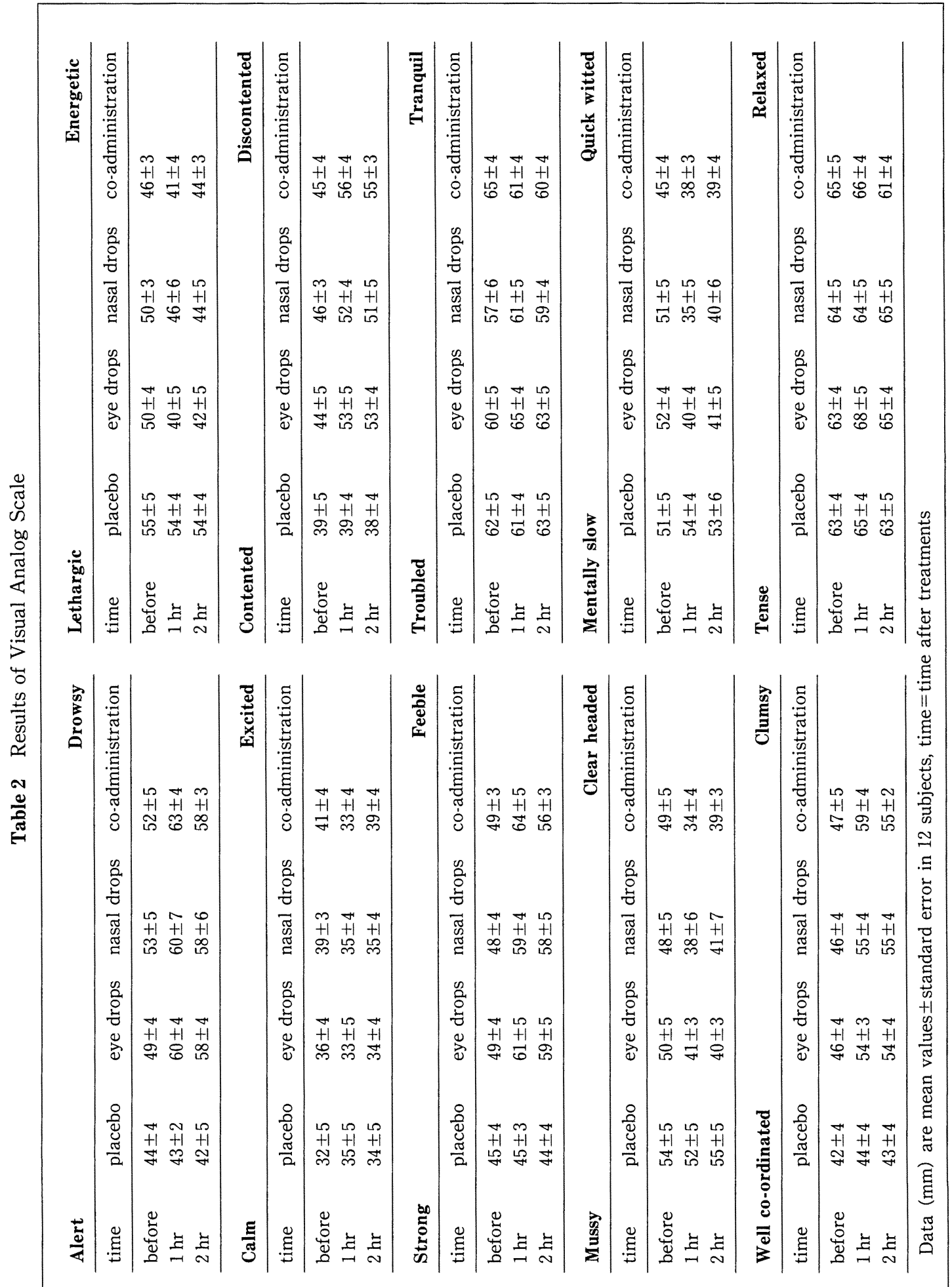




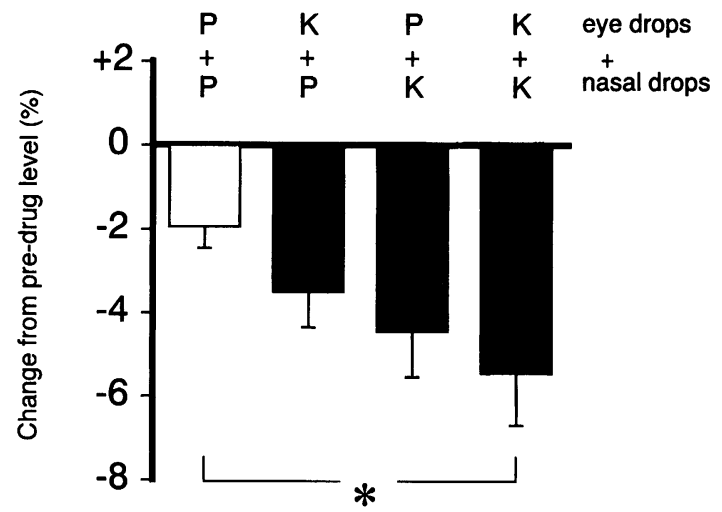

Fig. 1 Best effect on critical fusion frequency threshold (CFFT) after topical administration of ketotifen $(n=12)$

Significant $(\mathrm{p}<0.05)$ decreases in CFFT were observed after the co-administration of both eye drops and nasal drops of ketotifen. Bar height indicates mean-SE. Abbreviations : $\mathrm{K}=$ ketotifen, $\mathrm{P}=$ placebo. * : significant difference evaluated by Fisher's protected least significant difference test (one-tailed, $\mathrm{p}<0.05)$

ophthalmic and nasal solutions of ketotifen significantly reduced the frequency rate in CFFT $(\mathrm{p}<0.05)$ (Fig. 1). VAS showed no significant drowsiness, tiredness or sleepiness (Table 2).

\section{Discussion}

Ketotifen is a benzocycloheptathiphene derivative that possesses anti-allergic and histamine $\mathrm{H}_{1}$-receptor blocking effects. Mild and transient sedative effects are reportedly the main side effect associated with oral administration $^{11}$. As very low plasma concentrations are seen after topical administration compared with oral administration, it has been thought that ketotifen in small doses lacks any sedative effects. However, some outpatients on ketotifen eye drops have complained of sleepiness, and have asked for alternative antiallergic eye drops which have no sedative effect. This clinical experience prompted us to investigate whether topical ketotifen, given as eye drops and/or nasal drops, produces any effects on the central nervous system (CNS). We detected some CNS effects of ketotifen induced by the concomitant use of its ophthalmic and nasal solutions.

The drowsiness caused by anti-histamines is attributed to various mechanisms, including inhibition of the histamine $N$-methyltransferase and the blockage of central histaminergic receptors. Antagonism of other receptor connections with serotonergic, cholinergic, or central alphaadrenergic fibers may also be involved ${ }^{21}$. VAS was employed as a measure of the subjective effects of each treatment. On VAS there were no subjective changes in bodily feeling after topical medication. However, of the battery of psychomotor performance tests used to detect the CNS effects of topical ketotifen, CFFT detected the impairment of performance after ketotifen. CFFT is a means of measuring the ability to distinguish discrete sensory data and is taken as an index of the overall activity of CNS, suggesting that the impaired performance with ketotifen may be due to a nonspecific sedative effect ${ }^{3)}$. The sedative effect of ketotifen detected by CFFT in increasing order was: eye drops $<$ nasal drops $<$ eye drops + nasal drops.

The different degrees of the sedative effect of topical ketotifen may be explained by the amount of ketotifen absorbed by the different routes. In fact, post-marketing surveillance on the side-effects of ketotifen shows that the frequency of sleepiness was $0.80 \%$ for ocular, $1.03 \%$ for nasal and $4.41 \%$ for oral administration. Most of the eye drops instilled spill out of the eye, or are absorbed from the nasal mucus via the nasolacrimal duct ${ }^{4}$. There has been no report on the plasma concentrations of ketotifen following its topical administration, 
as the level is below that detected by HPLC. The plasma concentrations of ketotifen, after its co-administration via the two different topical routes, may exceed those after ocular or nasal administration alone.

The plasma concentration-time curve of timolol, a beta-adrenergic antagonist, following ocular or nasal administration shows that the Cmax of timolol given by the nasal route was four times higher than that by the ocular route ${ }^{5)}$. Although the plasma concentrations after topical administration are very low, timolol ophthalmic solution can impair respiratory function and exercise tolerance ${ }^{6}$. Severe bradycardia has been reported after concomitant use of verapamil, a calcium-channel antagonist, with topical timolol eye drops ${ }^{7,8}$. With atropine, the high systemic bioavailability of its $1 \%$ ophthalmic solution, which is $63.5 \pm 28.6 \%$, could explain its systemic anti-cholinergic side effects $^{9)}$.

The results of the present study appear to suggest a dose-effect or concentration-effect relationship of ketotifen. Recommended clinical doses of ketotifen, one or two drops four times a day for ophthalmic solution or one spray four times a day for nasal solution, will also produce higher concentrations in the steady state after a repeated dose regimen than those after a single dose as given in the present study. The sedative effect of consecutive oral ketotifen $1 \mathrm{mg}$ twice a day is the highest at the third day of one-week treatment. The highest sedative effect was proved by quantitative EEG, although none of the subjects studied felt sleepiness or tiredness ${ }^{1)}$. The detection of the relationship of reduced performance and the sedative action of drugs depends on the nature of the tests used and on their sensitivity to drugs ${ }^{10,11)}$.

Although the oral formulation of ketotifen is available in many countries, the topical formulation used in the present study is available only in Japan. Thus, our results suggest caution should be taken when the patient suffers from coexisting allergic diseases. There is the possibility that ketotifen might be prescribed by both in the treatment of patients with allergic conjunctivitis and rhinitis, especially in two different clinics. The concomitant topical use of ketotifen will impair daily performance which requires a high level of alertness. Further study is needed to evaluate whether topical ketotifen augments the sedative effects when it is used in combination with other oral drugs which are known to have a sedative effect or when it is used in a special patient population. As glucuronidation is involved in a major metabolic pathway of ketotifen ${ }^{12)}$, a drug interaction related to ketotifen drug metabolism is less likely.

In conclusion, the co-administration of ketotifen by two different topical routes, ocular and nasal, impaired the psychomotor performance in healthy men, suggesting that a dose-effect relationship of ketotifen may be involved.

\section{References}

1) Vollmer, R., Matejcek, M., Greenwood, C., et al. : Correlation between EEG changes indicative of sedation and subjective responses. Neuropsychobiology, $10: 249-253$ (1983).

2) Meltzer, E. O. : Antihistamine- and decongestantinduced performance decrements. Occup. Med., 32 : 327-334 (1990).

3) Nicholson, A. N. and Stone, B. M. : Antihistamines: impaired performance and the tendency to sleep. Eur. J. Clin. Pharmacol., 30:27-32 (1986).

4) Avan, G., Calissendorff, B., Seideman, P., et al. : Absorption of ocular timolol. Clin. Pharmacokinet., 5 : 95-100 (1980).

5) He, H., Edeki, T.I. and Wood, A. J. J. : Determination of low plasma timolol concentrations following topical application of timolol eye drops in humans by high performance liquid chromatography with electrochemical detection. J. 
Chromatogr. Biomed. Sci. Appl., 661 : 351-356 (1994).

6) Nelson, W. L., Fraunfelder, F. T., Sills, J. M., et al. : Adverse respiratory and cardiovascular events attributed to timolol ophthalmic solution, 1978-1985. Am. J. Ophthalmol., 102:606-611 (1986).

7) Pringle, S. D. : Severe bradycardia due to interaction of timolol eye drops and verapamil. BMJ, 294: 155-156 (1987).

8) Sinclair, N. I. and Benzie, J. L. : Timolol eye drops and verapamil-a dangerous combination. Med. J. Aust., 11 : 548 (1983).

9) Kaila, T., Korte, J.M. and Saari, K. M. : Systemic bioavailability of ocularly applied 1\% atropine. Acta. Ophthalmol. Scand., 77 : 193-196 (1999).

10) Hindmarch, I.: Psychomotor function and psychoactive drugs. Br. J. Clin. Pharmacol., 10 : 189-209 (1980).

11) Kerr, J. S., Dunmore, C. and Hindmarch, I. : The psychomotor and cognitive effects of a new antihistamine, mizolastine, compared to terfenadine, triprolidine and placebo in healthy volunteers. Eur. J. Clin. Pharmacol., 47 : 331-335 (1994).

12) Kennedy, G. R. : Metabolism and pharmacokinetics of ketotifen in children. Res. Clin. Forums, 4:17-20 (1982). 